\title{
Preserved Myocardial Deformation after Successful Coarctation Repair: A CMR Feature-Tracking Study
}

\author{
Elles J. Dijkema ${ }^{1}\left[\right.$ - Martijn G. Slieker ${ }^{1,2} \cdot$ Johannes M. P. J. Breur ${ }^{1} \cdot$ Tim Leiner $^{3} \cdot$ Heynric B. Grotenhuis $^{1}$
}

Received: 19 August 2017 / Accepted: 1 December 2017 / Published online: 5 December 2017

(c) The Author(s) 2017. This article is an open access publication

\begin{abstract}
Arterial vasculopathy and residual aortic obstruction can lead to left ventricular (LV) dysfunction in patients with coarctation of the aorta $(\mathrm{CoA})$ related to adverse ventriculo-arterial coupling. This study aimed to investigate potential differences in LV myocardial deformation indices between repaired CoA patients and healthy controls. Twenty-two CoA patients (age $30 \pm 10.6$ years) after surgical repair $(n=12)$ or balloon angioplasty (BA) $(n=10)$ without residual stenosis, between 3 months and 16 years of age with $>10$ years follow-up were compared to 22 healthy age- and gender-matched controls (age $30 \pm 3.8$ years). Cardiac magnetic resonance feature tracking (CMR-FT) was used for LV longitudinal-, circumferential-, and rotational deformation indices. Global systolic LV function was preserved in CoA patients (LV ejection fraction $58 \pm 4.8$ vs. $60 \pm 6.8 \%, p=0.56$ ) when compared to controls, with normal LV dimensions and mass $(p>0.05)$. Twelve CoA patients (55\%) were hypertensive, of whom 4 were on anti-hypertensive medication. LV global longitudinal strain was preserved in the four-chamber $(-18 \pm 4.4$ vs. $-16 \pm 4.7 \%, p=0.06)$ and two-chamber $(-22 \pm 5.1$ vs. $-20 \pm 6.0 \%, p=0.22)$ orientations in CoA patients. Global circumferential strain was preserved at basal $(-29 \pm 4.1$ vs. $-28 \pm 4.8 \%, p=0.43)$, mid-ventricular $(-27 \pm 4.2$ vs. $-25 \pm 3.0 \%, p=0.09)$, and apical levels $(-35 \pm 7.8$ vs. $-32 \pm 34.9 \%, p=0.32)$. No differences were found in global torsion $\left(2.4 \pm 1.3^{\circ}\right.$ vs. $\left.2.0 \pm 1.4^{\circ} \% \mathrm{~cm}, p=0.28\right)$, twist $\left(14 \pm 5.8^{\circ}\right.$ vs. $\left.12 \pm 6.3^{\circ}, p=0.34\right)$, and recoil rate $\left(-17 \pm 9.7^{\circ}\right.$ vs. $\left.-17 \pm 7.1^{\circ} \mathrm{cm} \mathrm{s}, p=0.97\right)$. Analysis of intra-observer variability demonstrated good reproducibility for all CMR deformation indices. Global and rotational myocardial deformation indices are preserved in CoA patients long-term after repair without residual stenosis, despite a high incidence of hypertension.
\end{abstract}

Keywords Coarctation of the aorta $\cdot$ Congenital heart disease $\cdot$ Feature tracking $\cdot$ Myocardial deformation

\section{Background}

Coarctation of the aorta (CoA) is defined as a localized narrowing of the aorta, which can be repaired through endovascular and/or surgical treatment [1,2]. Despite adequate relief of the stenosis, CoA patients still have a reduced life expectancy and an increased risk for cardiovascular complications including hypertension, left ventricular (LV) dysfunction,

Heynric B. Grotenhuis

H.B.Grotenhuis@umcutrecht.nl

1 Department of Pediatric Cardiology, Wilhelmina Children's Hospital, P.O. Box 85090, 3508 AB Utrecht, The Netherlands

2 Department of Pediatric Cardiology, Radboud University Medical Center, Nijmegen, The Netherlands

3 Department of Radiology, University Hospital Utrecht, Utrecht, The Netherlands atherosclerosis, and coronary artery- and cerebrovascular disease [3]. These long-term increased risks for morbidity and mortality suggest residual and/or progressive cardiovascular alterations long-term after CoA repair [3, 4]. Coarctation is associated with aortic vasculopathy, which may lead to increased stiffness of the arterial vascular bed [4]. Residual aortic obstruction in CoA patients is also associated with increased LV afterload and adverse ventricular-arterial coupling, ultimately resulting in LV myocardial dysfunction [5-10]. Whether adverse ventricular-arterial coupling is also present in well-repaired CoA patients is unclear.

Previous studies have demonstrated preserved or even increased global cardiac function, e.g., LV ejection fraction (LVEF), in patients treated for CoA [6, 11]. However, LVEF has been demonstrated to be a poor determinant of cardiovascular events as LVEF can remain within normal range despite ventricular dysfunction [11, 12]. Recent reports indicate that quantification of myocardial deformation 
provides a better insight in global and regional ventricular function, with a strong correlation to cardiovascular outcome [13-15]. Cardiovascular magnetic resonance imaging (CMR) is currently the preferred advanced imaging modality for non-invasive follow-up of congenital heart disease [2, 16]. Recently developed CMR feature-tracking (CMR-FT) software provides fast and accessible analysis of myocardial deformation of standard CMR cine images [17-19]. This study therefore aimed to investigate CMR-FT myocardial deformation indices of the LV in CoA patients long-term after repair without residual stenosis, with comparison between two treatment strategies: surgical repair and balloon angioplasty (BA).

\section{Methods}

\section{Study Population}

The study population consisted of 22 patients who were successfully treated for CoA (no signs of obstruction on echocardiographic imaging, arm-leg blood pressure gradient $<20 \mathrm{mmHg}$ ) with surgery or balloon angioplasty (BA) and had a follow-up of at least 10 years. Patients who underwent primary repair of localized membranous CoA between 3 months and 16 years of age between 1969 and 2004 in our institution were included in the study. To allow for comparison between surgery and BA, CoA patients with primary repair before 3 months of age were not included as surgical repair was routine for those patients. Patients with isthmus hypoplasia, aortic arch hypoplasia, and/or severe associated heart defects (e.g., hypoplastic left heart syndrome, transposition of the great arteries) were excluded. Twenty-two of the 72 included patients agreed to undergo CMR testing (inclusion rate: $31 \%$ ). Controls were recruited from a local cohort of healthy volunteers and were age- and gender matched to the CoA patient group.

\section{Imaging}

CMR imaging was performed on a $1.5 \mathrm{~T}$ scanner (Ingenia R4.1.2; Philips Healthcare, Best, The Netherlands). Cine images were obtained using a electrocardiogram-gated breath-hold balanced fast-field echo sequence (SSFP) in long-axis planes (two- and four-chamber views) and shortaxis slices covering the LV from the annulus of the mitral valve to the LV apex. Results for cardiac dimensions and LVEF were derived from short-axis slices according to the Society for Cardiovascular Magnetic Resonance recommendations [20]. End-diastolic LV mass, LV end-diastolic volume (EDV), and LV end-systolic volume (ESV) were indexed to body surface area (BSA) as described by Mosteller [21].

\section{CMR Analysis}

Cardiac magnetic resonance feature-tracking analysis was performed using feature-tracking software (2D Cardiac Performance Analysis MR 1.0; TomTec Imaging Systems, Unterschleissheim, Germany), based on tissue voxel motion tracking to analyze mechanical deformation [22]. Initial endo- and epicardial borders are drawn manually in the longitudinal and short-axis images [22]. The software subsequently assigns several characteristics (i.e., brightness, inhomogeneities, tissue patterns, and anatomic structures) to 48 tissue voxels in the initially set contour and tracks these voxels frame-by-frame using a hierarchical algorithm [22]. Movement of the features throughout the cardiac cycle enables derivation of deformation parameters such as strain and strain rate, as well as myocardial velocity and rotation [22]. Endocardial and epicardial borders were manually drawn in end-diastole with exclusion of papillary muscles in the endocardial border. The automatically propagated contour was assessed visually and adjustments were made manually in case of inappropriate tracking.

Longitudinal strain, strain rate, velocity, and displacement were measured in two- and four-chamber long-axis views (Fig. 1). Circumferential strain, strain rate, velocity, and displacement, as well as rotation and rotation rates were measured in short-axis views at basal (slice closest to the base with a full circle of myocardium), mid-ventricular (slice where the papillary muscles are free from the wall), and apical levels (apical slice showing LV cavity at endsystole, around $25 \% \mathrm{LV}$ distance, as this is has been shown to be the most appropriate apical location for analysis of rotational deformation [23]) (Fig. 2).

Myocardial deformation was analyzed in 16 myocardial segments, in accordance with the American Heart Association's standardized myocardial segmentation [10]. If adequate tracking of certain segments was not possible, these segments were excluded from analysis. Inadequate tracking was defined as a tracking deviation from the moving myocardial border $>50 \%$ of the myocardial width [18]. All myocardial deformation indices were measured three times in each patient in all views, and an average of all three measurements was used for results. Global longitudinal and circumferential strain was calculated as the average of the peak strain values of the subsequent segments.

Rotation was calculated in systole and diastole for the basal and apical slices, by averaging the rotation for all segments in the respective slice relative to the LV center axis. Positive rotation is considered counterclockwise when viewed from caudal to cranial position [22]. Torsion was calculated as the difference in apical (counterclockwise) and basal (clockwise) rotation divided by the 

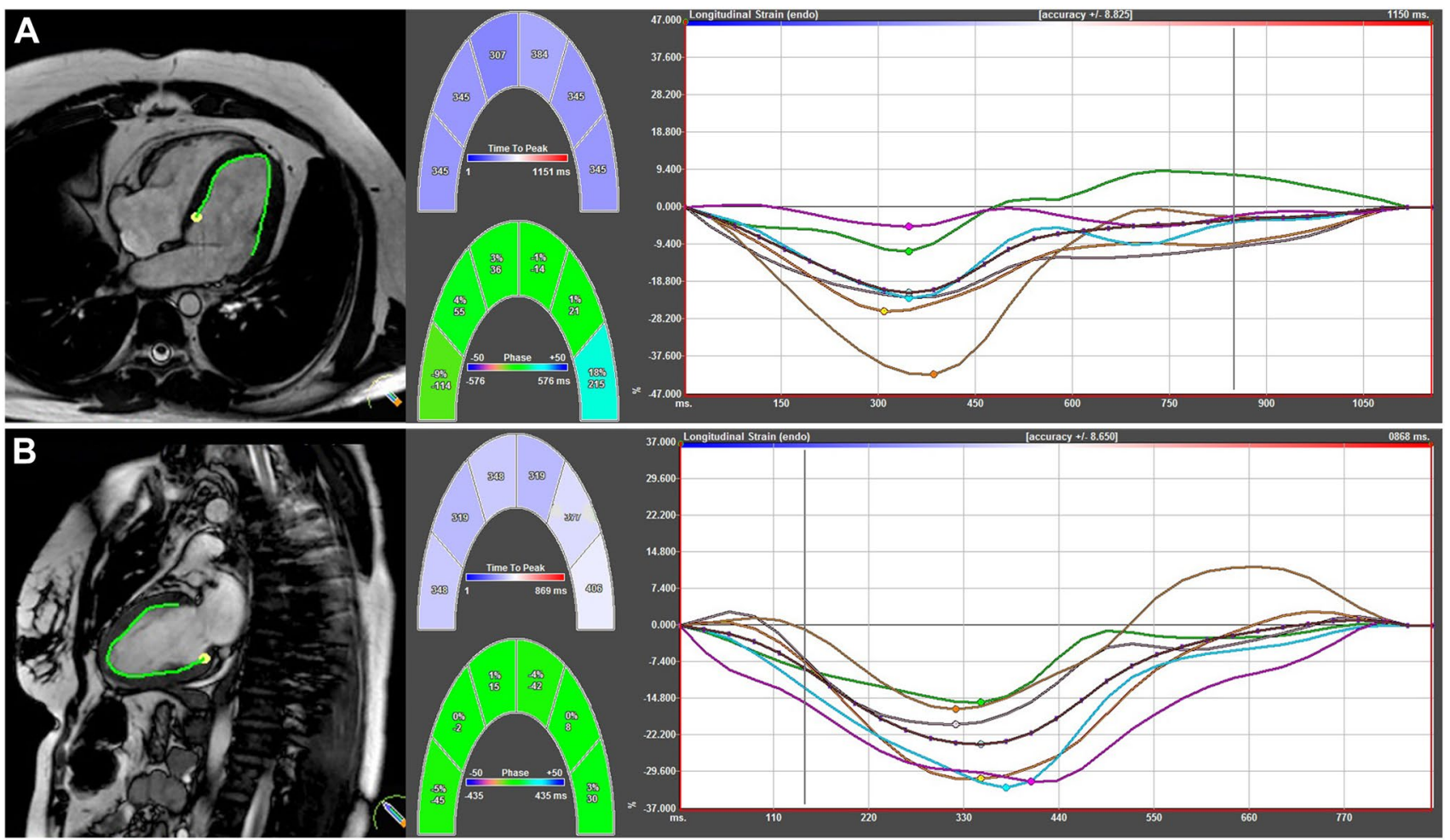

Fig. 1 Longitudinal myocardial strain analysis using CMR Feature tracking in a 4-chamber and b 2-chamber view. The left panels show endocardial tracking in 4- and 2-chamber views, resulting in horizon- tal (top) and vertical (bottom) strain curves. The coordinated curves with synchronized peak strain indicate preserved myocardial deformation inter-slice distance [23]. Calculation of torsion allows for comparison of twist deformation between patients with different cardiac sizes, especially applicable to the pediatric population [22]. Peak recoil rate was calculated as the maximum slope of the torsion-time curve as a measure of LV diastolic performance [22].

\section{Statistical Analysis}

Data are expressed as mean \pm standard deviation. Statistical comparisons of longitudinal-, and circumferential strain and strain rates, torsion and recoil rates between the CoA group, subgroups and normal controls were performed using unpaired Student's $t$ tests and one-way analysis of variance. Multiple regression was used to assess correlations between different cardiac variables and deformation indices. Intra-observer variability was assessed using the intraclass correlation coefficient (ICC). Data analyses were performed using SPSS, version 22 (International Business Machines Corporation, Armonk, New York, United States). A $p$ value $<0.05$ was considered statistically significant.

\section{Results}

The study population consisted of $22 \mathrm{CoA}$ patients and 22 healthy controls. Baseline characteristics of the study population are presented in Table 1. All CoA patients were primarily treated with surgery by end-to-end anastomosis (12 patients) or BA (10 patients). Mean age at intervention was 5.9 years (range $0.35-14.4$ years) and mean follow-up at the time of CMR was 23.9 years (range 10.8-42.4 years). Surgical patients were significantly older than BA patients $(35 \pm 9.5$ vs. $22 \pm 6.7$ years, $p<0.01)$, with a longer follow-up to CMR $(29 \pm 8.5$ vs. $18 \pm 2.9$ years, $p<0.01$ ), as BA is a more recently introduced technique in our practice. Systolic-, diastolic-, and mean arterial blood pressures (SBP, DBP, MAP) were $135 \pm 13.2$, $76 \pm 8.5$, and $96 \pm 8.8 \mathrm{mmHg}$, respectively, for CoA patients. Twelve CoA patients (55\%) were hypertensive according to the European Society of Hypertension Guidelines (SBP $\geq 95$ th percentile) [24]) at the time of investigation, of whom seven (58\%) had undergone surgery and five $(42 \%)$ BA. Five patients were using anti-hypertensive medication, of whom four were still hypertensive at the 

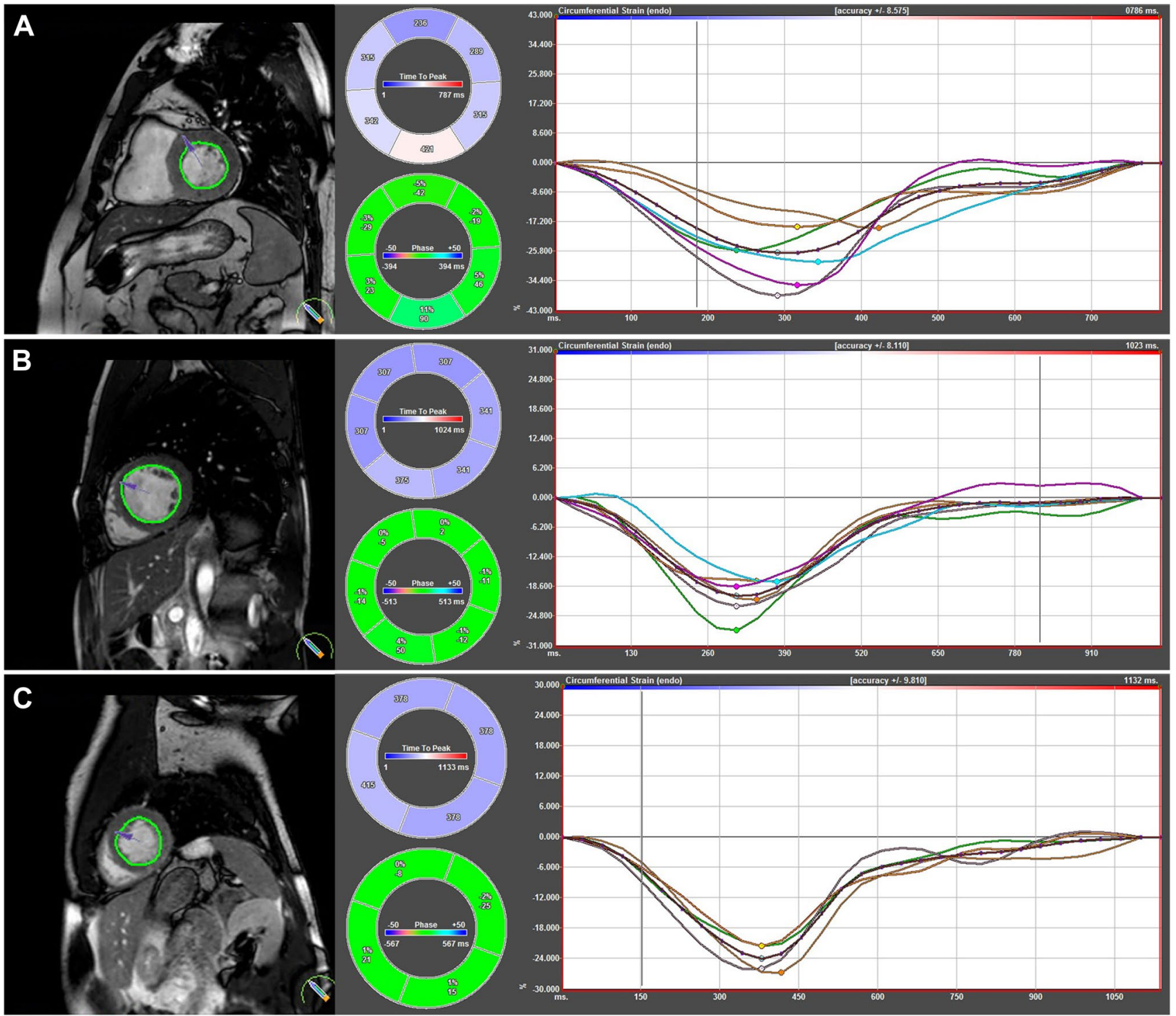

Fig. 2 Circumferential myocardial strain analysis using CMR Feature tracking in a basal, $\mathbf{b}$ mid-ventricular, and $\mathbf{c}$ apical sagittal view. The left panels show endocardial tracking in basal- (top), mid-ventricular-

time of investigation. None of the normotensive CoA patients were on anti-hypertensive medication. Nine patients $(41 \%)$ had a bicuspid aortic valve, none of which had significant valve dysfunction. Five patients (23\%), two BA and three surgical patients, had been successfully treated for re-CoA: two underwent surgery, one received BA, and two were treated with an endovascular stent. The median time between primary repair and re-CoA was 8.8 years (mean 13.8 years; range $2.1-40.4$ years). LV mass showed a significant positive correlation with age at time of repair $\left(R^{2}=0.78, p=0.046\right)$. No significant correlation was found between LV mass and age, blood pressures, time between repair and CMR, the repair to reintervention interval, or the reintervention to CMR interval (middle), and apical (bottom) views. The coordinaded curves with synchronized peak strain indicate preserved myocardial deformation

$\left(R^{2}=0.01-0.66, p>0.05\right)$. Systolic blood pressure was not significantly correlated with age, BSA, LV mass, intervention to CMR interval, age at time of repair, repair to reintervention interval, or reintervention to CMR interval $\left(R^{2}=0.03-0.69, p>0.05\right)$.

\section{Global Cardiac Function}

All CoA patients had preserved global systolic LV function when compared to controls (LVEF $59 \pm 4.8 \mathrm{vs.} 60 \pm 6.8 \%$, $p=0.56$ ) (Table 2). LV mass, LVEDV, and LVESV were also normal in CoA patients ( $p \geq 0.05$ for all). No significant difference in LVEF was found between patients treated 
Table 1 Baseline characteristics of the study population

\begin{tabular}{llll}
\hline & CoA & Control & $p$ value \\
& $n=22$ & $n=22$ & \\
\hline Age & $30 \pm 10.6$ & $30 \pm 3.8$ & 0.87 \\
BSA & $1.9 \pm 0.22$ & $2.0 \pm 0.12$ & 0.81 \\
MAP & $96 \pm 8.8$ & $94.8 \pm 8.5$ & 0.72 \\
SBPday & $135 \pm 13.2$ & $129 \pm 10.8$ & 0.12 \\
HR & $65 \pm 11.0$ & $60 \pm 9.7$ & 0.11 \\
Intervention & & & \\
$\quad$ Surgery & $12(55 \%)$ & - & \\
BA & $10(45 \%)$ & - & \\
Hypertension & $12(55 \%)$ & - & \\
BAV & $9(41 \%)$ & - & \\
Reintervention & $5(23 \%)$ & - & \\
\hline
\end{tabular}

$C O A$ coarctation of the aorta, BSA body surface area, MAP mean arterial pressure, SBPday systolic blood pressure during daytime, $H R$ heart rate, $B A$ balloon angioplasty, $B A V$ bicuspid aortic valve

for re-CoA and patients without re-CoA (LVEF 59 $\pm 3.9 \mathrm{vs}$. $59 \pm 5.1 \%, p=0.75)$.

\section{Left Ventricular Myocardial Deformation}

LV global longitudinal strain in the four-chamber and twochamber planes was similar between CoA patients and controls ( $p=0.06$ and $p=0.22$, respectively). Global circumferential strain was also preserved at basal $(p=0.43)$, mid-ventricular $(p=0.09)$, and apical levels $(p=0.32)$. Global longitudinal strain rate was increased in CoA patients when compared to controls (four-chamber: $-0.8 \pm 0.23$ vs. $-0.7 \pm 0.22 \%, p<0.05$; two-chamber: $-1.1 \pm 0.31$ vs. $-0.8 \pm 0.30 \%, p<0.05)$. Global torsion $(p=0.28)$, twist $(p=0.34)$, and recoil rate $(p=0.97)$ demonstrated no differences between CoA patients and controls $(p=0.28,0.34$ and 0.97 , respectively).

Global longitudinal strain was significantly correlated with LVEF $\left(R^{2}=0.234, p<0.01\right)$, and global circumferential strain was significantly correlated with LVEF at the mid-ventricular and apical levels $\left(R^{2}=0.01, p=0.01\right.$ and $R^{2}=0.09, p=0.047$, respectively). Basal circumferential strain showed a significant correlation with age at the time of repair $\left(R^{2}=0.29, p<0.01\right)$. None of the studied LV myocardial deformation indices correlated with SBP, DPB, and $\operatorname{MAP}\left(R^{2}=0.001-0.156, p>0.05\right.$ for all indices $)$.

\section{Balloon Angioplasty Compared to Surgery}

Patients treated with BA were significantly younger at time of investigation $(22 \pm 6.7$ vs. $36 \pm 9.5$ years, $p<0.01)$ and showed a slightly higher LVEF $(61 \pm 4.0$ vs. $57 \pm 4.7 \%$, $p<0.05)$ when compared to CoA patients treated with
Table 2 Global left ventricular (LV) function and LV deformation in coarctation versus healthy controls

\begin{tabular}{|c|c|c|c|}
\hline & $\begin{array}{l}\mathrm{CoA} \\
n=22\end{array}$ & $\begin{array}{l}\text { Controls } \\
n=22\end{array}$ & $p$ value \\
\hline LVEF & $59 \pm 4.77$ & $60 \pm 6.84$ & 0.56 \\
\hline LV mass & $68 \pm 8.49$ & $64 \pm 8.63$ & 0.08 \\
\hline EDV & $93 \pm 15.2$ & $94 \pm 9.3$ & 0.79 \\
\hline ESV & $26 \pm 7.0$ & $31 \pm 7.3$ & 0.05 \\
\hline \multicolumn{4}{|l|}{ Strain } \\
\hline \multicolumn{4}{|l|}{ GLS } \\
\hline Horizontal & $-18.5 \pm 4.37$ & $-15.8 \pm 4.69$ & 0.06 \\
\hline Vertical & $-21.8 \pm 5.10$ & $-19.7 \pm 6.04$ & 0.22 \\
\hline \multicolumn{4}{|l|}{ GCS } \\
\hline Basal & $-29.3 \pm 4.06$ & $-28.2 \pm 4.79$ & 0.43 \\
\hline $\mathrm{Mv}$ & $-27.0 \pm 4.19$ & $-25.1 \pm 2.96$ & 0.09 \\
\hline Apex & $-34.6 \pm 7.79$ & $-32.6 \pm 4.88$ & 0.32 \\
\hline \multicolumn{4}{|l|}{ Strain rate } \\
\hline \multicolumn{4}{|l|}{ GLSR } \\
\hline Horizontal & $-0.8 \pm 0.23$ & $-0.7 \pm 0.22$ & 0.02 \\
\hline Vertical & $-1.1 \pm 0.31$ & $-0.8 \pm 0.30$ & 0.02 \\
\hline \multicolumn{4}{|l|}{ GCSR } \\
\hline Base & $-1.5 \pm 0.30$ & $-1.4 \pm 0.31$ & 0.25 \\
\hline $\mathrm{Mv}$ & $-1.4 \pm 0.31$ & $-1.3 \pm 0.25$ & 0.33 \\
\hline Apex & $-1.9 \pm 0.47$ & $-1.7 \pm 0.32$ & 0.07 \\
\hline \multicolumn{4}{|l|}{ Rotation } \\
\hline Twist & $12.3 \pm 6.29$ & $14.2 \pm 5.83$ & 0.33 \\
\hline Torsion & $2.0 \pm 1.35$ & $2.4 \pm 1.35$ & 0.28 \\
\hline Recoil rate & $-17.8 \pm 7.11$ & $-17.7 \pm 9.73$ & 0.97 \\
\hline
\end{tabular}

LV mass, LVEDV, and LVESV are indexed to body surface area

$C o A$ coarctation of the aorta, $L V E F L V$ ejection fraction, $L V E D V$ enddiastolic volume, $L V E S V$ end-systolic volume, MAP mean arterial pressure, SBPday mean systolic blood pressure during daytime, $H R$ heart rate, GLS global longitudinal strain, GCS global circumferential strain, GLSR global longitudinal strain rate, GCSR global circumferential strain rate

surgery. Two surgical patients had an LVEF below 55\% (48 and $49 \%$, respectively), versus one BA patient (54\%). No CoA patient had an LVEF $<40 \%$. Global strain and rotational indices between the two patient groups were comparable $(p>0.05)$, despite a slightly decreased apical circumferential strain rate in surgical CoA patients $(-1.7 \pm 0.47$ vs. $-2.1 \pm 0.38 / \mathrm{s}, p<0.05)$. No significant differences in $\operatorname{SBP}(136 \pm 13$ vs. $133 \pm 14 \mathrm{mmHg}, p=0.66)$, DBP $(75 \pm 4$ vs. $78 \pm 12 \mathrm{mmHg}, p=0.56)$, and MAP (96 \pm 6 vs. $96 \pm 12 \mathrm{mmHg}, p=0.88$ ) were found between surgical and BA patients.

\section{Hypertension}

Hypertensive CoA patients (with $\mathrm{SBP} \geq 95$ th percentile according to the European Society of Hypertension 
Guideline [24]) demonstrated a similar LV mass when compared to normotensive CoA patients or controls $(68 \pm 8.9 \mathrm{vs}$. $67 \pm 8.4 \mathrm{~g} / \mathrm{m}^{2}$ and $\left.64 \pm 8.6 \mathrm{~g} / \mathrm{m}^{2}, p=0.20\right)$ and a similar LV mass to LVEDV ratio $(0.83 \pm 0.14$ vs. $0.90 \pm 0.18 \mathrm{~g} / \mathrm{ml}$ and $0.85 \pm 0.15 \mathrm{~g} / \mathrm{ml}, p=0.59)$. No significant differences were found for LVEF ( $p=0.83$ ) or LV dimensions ( $p=0.62$ and 0.09 for LVEDV and LVESV, respectively). Hypertensive CoA patients demonstrated a slightly increased global longitudinal strain in the four-chamber plane when compared to normotensive patients and controls $(-19.4 \pm 3.33$ vs. $-17.4 \pm 5.34 \%$ and $-15.8 \pm 4.69 \%$, respectively, $p=0.10)$,

Table 3 Global left ventricular (LV) function and LV deformation in normotensive- and hypertensive CoA patients and controls

\begin{tabular}{|c|c|c|c|c|}
\hline & $\begin{array}{l}\text { Controls } \\
n=22\end{array}$ & $\begin{array}{l}\text { CoA NT } \\
n=10\end{array}$ & $\begin{array}{l}\text { CoA HT } \\
n=12\end{array}$ & $p$ \\
\hline LVEF & $60 \pm 6.8$ & $59 \pm 6.3$ & $58 \pm 3.3$ & 0.83 \\
\hline LV mass & $63 \pm 8.6$ & $67 \pm 8.4$ & $68 \pm 8.9$ & 0.21 \\
\hline EDV & $94 \pm 8.6$ & $90 \pm 13.3$ & $96 \pm 16.7$ & 0.62 \\
\hline ESV & $31 \pm 7.3$ & $24 \pm 6.9$ & $27 \pm 7.1$ & 0.09 \\
\hline MAP & $94.8 \pm 8.5$ & $89 \pm 4.2$ & $101 \pm 8.0$ & $<0.01$ \\
\hline SBPday & $129 \pm 10.8$ & $123 \pm 6.4$ & $144 \pm 9.3$ & $<0.01$ \\
\hline HR & $60 \pm 9.7$ & $62 \pm 10.8$ & $68 \pm 10.8$ & 0.12 \\
\hline \multicolumn{5}{|l|}{ Strain } \\
\hline \multicolumn{5}{|l|}{ GLS } \\
\hline Horizontal & $-15.8 \pm 4.69$ & $-17.4 \pm 5.34$ & $-19.4 \pm 3.33$ & 0.10 \\
\hline Vertical & $-19.7 \pm 6.04$ & $-22.8 \pm 5.06$ & $-21.1 \pm 5.24$ & 0.38 \\
\hline \multicolumn{5}{|l|}{ GCS } \\
\hline Basal & $-28.2 \pm 4.79$ & $-29.7 \pm 4.97$ & $-28.9 \pm 3.31$ & 0.68 \\
\hline $\mathrm{Mv}$ & $-25.1 \pm 2.96$ & $-27.7 \pm 2.62$ & $-26.4 \pm 5.19$ & 0.17 \\
\hline Apex & $-32.6 \pm 4.88$ & $-35.1 \pm 8.01$ & $-34.2 \pm 7.93$ & 0.58 \\
\hline \multicolumn{5}{|l|}{ Strain rate } \\
\hline \multicolumn{5}{|l|}{ Glsr } \\
\hline Horizontal & $-0.7 \pm 0.22$ & $-0.8 \pm 0.25$ & $-0.8 \pm 0.21$ & 0.07 \\
\hline Vertical & $-0.8 \pm 0.30$ & $-1.1 \pm 0.31$ & $-1.0 \pm 0.33$ & 0.07 \\
\hline \multicolumn{5}{|l|}{ GCSR } \\
\hline Base & $-1.4 \pm 0.31$ & $-1.5 \pm 0.31$ & $-1.5 \pm 0.32$ & 0.51 \\
\hline $\mathrm{Mv}$ & $-1.3 \pm 0.25$ & $-1.4 \pm 0.21$ & $-1.4 \pm 0.37$ & 0.48 \\
\hline Apex & $-1.7 \pm 0.32$ & $-1.9 \pm 0.49$ & $-1.9 \pm 0.48$ & 0.17 \\
\hline \multicolumn{5}{|l|}{ Rotation } \\
\hline Twist & $12.3 \pm 6.29$ & $14.9 \pm 5.92$ & $13.6 \pm 5.95$ & 0.55 \\
\hline Torsion & $2.0 \pm 1.35$ & $2.7 \pm 1.64$ & $2.2 \pm 1.07$ & 0.34 \\
\hline Recoil rate & $-17.8 \pm 7.11$ & $-19.7 \pm 12.71$ & $-16.0 \pm 6.45$ & 0.60 \\
\hline
\end{tabular}

LV mass, LVEDV and LVESV are indexed to body surface area

$C O A$ coarctation, $H T$ hypertensive patients, $N T$ normotensive patients, $C o$ controls, $L V E F$ LV ejection fraction, $E D V L V$ end-diastolic volume, $E S V L V$ end-systolic volume, $M A P$ mean arterial pressure, $S B P$ day mean systolic blood pressure during daytime, $H R$ heart rate, $G L S$ global longitudinal strain, GCS global circumferential strain, GLSR global longitudinal strain rate, GCSR global circumferential strain rate

$p$ values are given in the three most right columns but this difference was not statistically significant (Table 3). No significant differences were found in strain rate or rotational indices between hypertensive CoA patients, normotensive patients, and controls.

\section{Reproducibility}

CMR-FT demonstrated good intra-observer reproducibility, with an intraclass correlation coefficient $>0.8$ for all global strain, strain rate, and rotational indices. All ICCs are presented in Table 4.

\section{Discussion}

Repaired CoA patients demonstrate an increased susceptibility for cardiovascular morbidity and mortality at longterm follow-up [9, 10, 25-27]. Residual aortic obstruction in CoA patients is associated with adverse ventricular-arterial coupling related to increased LV afterload [10]. Whether a similar cascade is also present in well-repaired CoA patients is unknown. The present study quantifies myocardial deformation in CoA patients long-term after surgical or BA repair without residual stenosis. This study adds the following to our understanding of cardiovascular health in well-repaired CoA patients long-term after repair:

- global LV systolic function is generally normal;

- global and regional myocardial deformation indices are preserved in well-repaired CoA patients, despite a high incidence of hypertension;

- the choice for treatment modality - surgery versus BAhas no impact on LV systolic function or LV myocardial deformation.

Table 4 The intraclass correlation coefficients (ICC) for global myocardial deformation measurements

\begin{tabular}{llll}
\hline Strain & & ICC & $95 \%$ CI \\
\hline GLS & Horizontal & 0.939 & $(0.875-0.973)$ \\
& Vertical & 0.927 & $(0.850-0.968)$ \\
GCS & Basal & 0.952 & $(0.903-0.979)$ \\
& Mid-ventricular & 0.945 & $(0.890-0.976)$ \\
& Apical & 0.980 & $(0.958-0.991)$ \\
Strain rate & & & \\
GLSR & Horizontal & 0.859 & $(0.716-0.937)$ \\
& Vertical & 0.925 & $(0.846-0.967)$ \\
GCSR & Basal & 0.964 & $(0.928-0.984)$ \\
& Mid-ventricular & 0.985 & $(0.970-0.993)$ \\
& Apical & 0.938 & $(0.875-0.972)$ \\
\hline
\end{tabular}

GLS global longitudinal strain, GCS global circumferential strain, GLSR global longitudinal strain rate, GCSR global circumferential strain rate 


\section{Global Cardiac Function}

Global LV function was preserved in our cohort of wellrepaired CoA patients at long-term follow-up as previously described, expressed by normal LVEF $[4,9,12,26]$. Our CoA cohort also demonstrated normal LV mass and LV dimensions, suggesting that no significant LV remodeling related to potentially increased $\mathrm{LV}$ afterload is present in well-repaired CoA patients, which is consistent with other CMR and echocardiographic reports $[9,10,26]$. In contrast, several echocardiographic studies showed increased LV mass after CoA repair, although these studies included CoA patients treated at significantly older age or with a longer follow-up duration ( $>20$ years) $[4,11,25]$. Increased LV mass is thought to be the result of increased afterload and has been related to residual aortic stenosis at the site of previous repair [26]. Our study demonstrates that repaired CoA patients without residual stenosis not only have preserved LV systolic function by LVEF, but also normal myocardial mass.

\section{Left Ventricular Myocardial Deformation}

Myocardial deformation was preserved in our CoA patients when compared to healthy controls, in agreement with previous echocardiography and CMR-FT reports indicating preserved longitudinal and circumferential strain in $\mathrm{CoA}$ patients with preserved global LV systolic function and normal LV mass $[9,11]$. Jashari et al. found reduced LV global longitudinal strain and strain rate prior to surgical CoA repair, with a further decrease shortly after intervention [28]. Interestingly, progressive normalization of strain was observed over 2 years after satisfactory repair, resulting in near-normal LV longitudinal strain values [28]. Kowalski et al. also showed normal global LV function and LV longitudinal strain in CoA patients by $2 \mathrm{D}$ echocardiographic deformation 10 years after surgical repair, suggesting a full recovery of the myocardium at long-term follow-up [11]. In contrast, a recent CMR-FT study by Shang et al. showed impaired longitudinal deformation in CoA patients treated in the neonatal period [10]. This study included well-repaired CoA patients treated with surgery, BA, or stent placement at an early age (before 3 months of age) [10]. In the current study, patients treated before 3 months of age were excluded, as most patients treated at this young age suffer from a more severe coarctation, requiring immediate correction. Abnormal longitudinal deformation was explained by Shang et al. by increased arterial stiffness and altered ventriculo-arterial coupling [10]. The pulse wave velocity in the proximal aorta was increased with increased aortic stiffness, resulting in an early return of the reflected wave, imposing increased afterload and impaired LV relaxation [10].
Surprisingly, our CoA patients demonstrated increased systolic global longitudinal strain rate when compared to our control group. Systolic strain rate has been identified as a strong indicator for LV contractility. Increased contractile performance has been described in CoA patients after repair, as CoA patients experience increased afterload which may lead to myocardial remodeling and LV hypertrophy to maintain normal LV wall stress levels [29]. Kimball et al. suggested that repair of coarctation may relieve the increased afterload, but myocardial hypertrophy may partially persist which results in a hypercontractile myocardial state after repair. In addition, GLS demonstrated a trend towards lower GLS in controls, which may reflect the same phenomenon of a hypercontractile state in the CoA patient group.

Conflicting data on myocardial deformation after CoA repair may be explained by differences in age at the time of repair, associated severity of $\mathrm{CoA}$, and duration of follow-up between studies. The myocardial response to increased pressure load is age-dependent as the myocardium has the ability to regenerate and increase vascularity in children, thereby restoring pressure-induced remodeling with preservation of coronary blood flow and reduction of development of myocardial fibrosis $[12,30]$. Delayed repair of CoA may induce a maladaptive response of the LV, resulting in adverse outcome later in life [12]. Age at the time of repair has therefore been identified as a predictor for impaired longitudinal strain [31]. In addition, the set point of the renin-angiotensin-aldosterone system is defined in neonatal life [32]. Delayed CoA repair may therefore lead to more abnormal development of the renin-angiotensin-aldosterone system, which may in turn lead to hypertension and concomitant increased LV afterload [33, 34]. This study showed a significant correlation between age at time of repair and LV mass. However, we found no influence of age at time of repair on longitudinal strain in patients with mild-to-moderate $\mathrm{CoA}$ treated at a mean age of 5.9 years. Preserved longitudinal strain might suggest limited effects of adverse arterial-ventricular coupling in this patient group long-term after successful CoA repair. However, as assessment of aortic vascular function was not performed in this study, we can not assess the influence of arterial stiffness.

Jashari et al. reported more impaired longitudinal strain in CoA patients with LV hypertrophy, as confirmed by other studies $[9,27,28,31]$. The myofiber orientation changes throughout the myocardium, with a predominantly longitudinal orientation in the endomyocardium [10,27]. The endomyocardial fibers are most sensitive to pressure load-induced fibrosis [27]. Therefore, longitudinal strain is primarily affected in patients with pressure load-induced LV hypertrophy and LV myocardial dysfunction [27]. Well-repaired CoA patients in our cohort showed normal LV mass-hence the absence of compensatory LV hypertrophy - which may explain the preserved LV deformation 
indices. Our findings of preserved myocardial deformation after coarctation repair in the absence of LV hypertrophy are in line with a previous report of Kutty et al. [9], who demonstrated reduced longitudinal LV strain in CoA patients with increased LV mass and preserved longitudinal strain in patients with normal LV mass by use of CMR-FT [9]. In contrast to the study by Kutty et al. our current study also assessed the influence of hypertension on myocardial deformation. Our results demonstrate preserved myocardial deformation even in hypertensive patients in absence of LV hypertrophy, which might suggest that LV myocardial dysfunction occurs at a later stage in the cascade of arterial-ventricular coupling.

No difference in myocardial deformation was observed between CoA patients after BA and surgery in our study. Patients after surgery had slightly lower LVEF compared to BA patients, possibly due to the older age of the surgical patients. Similar results have been described by Chui et al. who showed a minor-non-significant-difference in LVEF between both treatment groups [35].

\section{Hypertension}

Hypertension is a well-described long-term complication of $\mathrm{CoA}[3,22,36]$. Hypertension is associated with an increased afterload, LV hypertrophy, and myocardial fibrosis [27]. Despite a high prevalence of hypertension in our patient group, LV mass and LV dimensions were preserved in our CoA patients. Hypertension did not affect global LV function or myocardial deformation in our cohort, suggesting that hypertension does not significantly affect cardiac function of well-repaired CoA patients at this point in follow-up. In fact, hypertensive CoA patients seemed to have slightly increased strain compared to controls. This difference is most likely not clinically relevant, as both strain values fall within normal range [17]. Left ventricular remodeling and hypertrophy with impaired LV deformation have been described in hypertensive patients [37-41]. In contrast to our current findings, Shang et al. reported increased LV mass and decreased myocardial deformation in hypertensive CoA patients compared to healthy controls. However, in accordance to our results, LV mass and myocardial deformation did not differ significantly between normotensive and hypertensive CoA patients [10]. Indeed, increased systolic blood pressure has been associated with concentric hypertrophy (increased LV mass combined with increased relative wall thickness) [42]. However, development of myocardial concentric hypertrophy is a gradual progressive process, which is usually preceded by concentric remodeling in which the relative wall thickness is increased, but LV mass is preserved [42].

\section{Limitations}

A few limitations should be addressed regarding this study. The relatively small sample size limits the generalizability of the results and may have obscured further differences and associations. Our CoA patients were not analyzed for arterial stiffness, so direct assessment of ventriculo-arterial coupling could not be performed. Nevertheless, this study provides insight in myocardial deformation in CoA patients at long-term follow-up, suggesting good LV function in well-repaired patients. The long-term implications of our findings need to be substantiated with follow-up studies.

\section{Conclusions}

Feature tracking by CMR provides easily accessible and reproducible analysis of myocardial deformation. Wellrepaired CoA patients show preserved cardiac function by normal LV ejection fraction and normal myocardial deformation indices at long-term follow-up, despite a high incidence of hypertension. We found good reproducibility for strain, strain rate, and rotational indices with CMR-FT, suggesting CMR-FT is a useful tool for analysis of myocardial deformation.

Acknowledgements This study was funded by a grant from the "Stichting Hartekind".

\section{Compliance with Ethical Standards}

Conflict of interest The authors declare that they have no conflict of interest.

Ethical Approval All authors state that this study complies with the Declaration of Helsinki.

Open Access This article is distributed under the terms of the Creative Commons Attribution 4.0 International License (http://creativecommons.org/licenses/by/4.0/), which permits unrestricted use, distribution, and reproduction in any medium, provided you give appropriate credit to the original author(s) and the source, provide a link to the Creative Commons license, and indicate if changes were made.

\section{References}

1. Baumgartner H, Bonhoeffer P, De Groot NM, de Haan F, Deanfield JE, Galie N, Gatzoulis MA, Gohlke-Baerwolf C, Kaemmerer H, Kilner P, Meijboom F, Mulder BJ, Oechslin E, Oliver JM, Serraf A, Szatmari A, Thaulow E, Vouhe PR, Walma E (2010) Task Force on the Management of Grown-up Congenital Heart Disease of the European Society of Cardiology (ESC); Association for 
European Paediatric Cardiology (AEPC); ESC Committee for Practice Guidelines (CPG). ESC Guidelines for the management of grown-up congenital heart disease (new version 2010). Eur Heart J 31:2915-2957

2. Karaosmanoglu AD, Khawaja RD, Onur MR, Kalra MK (2015) CT and MRI of aortic coarctation: pre- and postsurgical findings. AJR Am J Roentgenol 204(3):224-233

3. Canniffe C, Ou P, Walsh K, Bonnet D, Celermajer D (2013) Hypertension after repair of aortic coarctation-a systematic review. Int J Cardiol 167(6):2456-2461

4. Li VW, Cheung YF (2015) Arterial-left ventricular-left atrial coupling late after repair of aortic coarctation and interruption. Eur Heart J Cardiovasc Imaging 16(7):771-780

5. Kenny D, Polson JW, Martin RP, Paton JFR, Wolf AR (2011) Hypertension and coarctation of the aorta: an inevitable consequence of developmental pathophysiology. Hypertens Res 34(5):543-547

6. O'Sullivan J (2014) Late hypertension in patients with repaired aortic coarctation. Curr Hypertens Rep 16(3):421

7. Frydrychowicz A, Markl M, Hirtler D, Harloff A, Schlensak C, Geiger J, Stiller B, Arnold R (2011) Aortic hemodynamics in patients with and without repair of aortic coarctation: in vivo analysis by $4 \mathrm{D}$ flow-sensitive magnetic resonance imaging. Invest Radiol 46(5):317-325

8. Lee JJ, D'Ancona G, Amaducci A, Follis F, Pilato M, Pasta S (2014) Role of computational modeling in thoracic aortic pathology: a review. J Card Surg 29(5):653-662

9. Kutty S, Rangamani S, Venkataraman J, Li L, Schuster A, Fletcher SE, Danford DA, Beerbaum P (2013) Reduced global longitudinal and radial strain with normal left ventricular ejection fraction late after effective repair of aortic coarctation: a CMR feature tracking study. Int J Cardiovasc Imaging 29(1):141-150

10. Shang Q, Sarikouch S, Patel S, Schuster A, Steinmetz M, Ou P, Danford DA, Beerbaum P, Kutty S (2016) Assessment of ventriculo-vascular properties in repaired coarctation using cardiac magnetic resonance-derived aortic, left atrial and left ventricular strain. Eur Radiol 27:167-177

11. Kowalski M, Kowalik E, Kotliński K, Hoffman P (2009) Regional left ventricular myocardial shortening in normotensive patients late after aortic coarctation repair-normal or impaired? Ultrasound Med Biol 35(12):1947-1952

12. Van der Ende J, Vázquez Antona CA, Erdmenger Orellana J, Romero Cárdenas Á, Roldan FJ, Vargas Barrón J (2013) Left ventricular longitudinal strain measured by speckle tracking as a predictor of the decrease in left ventricular deformation in children with congenital stenosis of the aorta or coarctation of the aorta. Ultrasound Med Biol 39(7):1207-1214

13. Smith BM, Dorfman AL, Yu S, Russell MW, Agarwal PP, Ghadimi Mahani M, Lu JC (2014) Relation of strain by feature tracking and clinical outcome in children, adolescents, and young adults with hypertrophic cardiomyopathy. Am J Cardiol 114(8):1275-1280

14. Buss SJ, Breuninger K, Lehrke S, Voss A, Galuschky C, Lossnitzer D, Andre F, Ehlermann P, Franke J, Taeger T, Frankenstein L, Steen H, Meder B, Giannitsis E, Katus HA, Korosoglou $\mathrm{G}$ (2015) Assessment of myocardial deformation with cardiac magnetic resonance strain imaging improves risk stratification in patients with dilated cardiomyopathy. Eur Heart J Cardiovasc Imaging 16(3):307-315

15. Rangarajan V, Chacko SJ, Romano S, Jue J, Jariwala N, Chung J, Farzaneh-Far A (2016) Left ventricular long axis function assessed during cine-cardiovascular magnetic resonance is an independent predictor of adverse cardiac events. J Cardiovasc Magn Reson 18(1):35

16. Holloway BJ, Rosewarne D, Jones RG (2011) Imaging of thoracic aortic disease. Br J Radiol 84(3):S338-S354
17. Andre F, Steen H, Matheis P, Westkott M, Breuninger K, Sander Y, Kammerer R, Galuschky C, Giannitsis E, Korosoglou G, Katus HA, Buss SJ (2015) Age- and gender-related normal left ventricular deformation assessed by cardiovascular magnetic resonance feature tracking. J Cardiovasc Magn Reson 17:25

18. Augustine D, Lewandowski AJ, Lazdam M, Rai A, Francis J, Myerson S, Noble A, Becher H, Neubauer S, Petersen SE, Leeson P (2013) Global and regional left ventricular myocardial deformation measures by magnetic resonance feature tracking in healthy volunteers: comparison with tagging and relevance of gender. J Cardiovasc Magn Reson 15:8

19. Aurich M, Keller M, Greiner S, Steen H, Aus dem Siepen F, Riffel J, Katus HA, Buss SJ, Mereles D (2016) Left ventricular mechanics assessed by two-dimensional echocardiography and cardiac magnetic resonance imaging: comparison of high-resolution speckle tracking and feature tracking. Eur Heart J Cardiovasc Imaging 12:jew042

20. Schulz-Menger J, Bluemke D, Bremerich J, Flamm SD, Fogel MA, Friedrich MG, Kim RJ, von Knobelsdorff-Brenkenhoff F, Kramer CM, Pennell DJ, Plein S, Nagel E (2013) Standardized image interpretation and post processing in cardiovascular magnetic resonance: Society for Cardiovascular Magnetic Resonance (SCMR) Board of Trustees Task Force on Standardized Post Processing. J Cardiovasc Magn Reson 15:35

21. Mosteller RD (1987) Simplified calculation of body surface area. New Engl J Med 317:1098

22. Hor KN, Baumann R, Pedrizzetti G, Tonti G, Gottliebson WM, Taylor M, Benson DW, Mazur W (2011) Magnetic resonance derived myocardial strain assessment using feature tracking. J Vis Exp 48:2356

23. Kowallick JT, Lamata P, Hussain ST, Kutty S, Steinmetz M, Sohns JM, Fasshauer M, Staab W, Unterberg-Buchwald C, Bigalke B, Lotz J, Hasenfuß G, Schuster A (2014) Quantification of left ventricular torsion and diastolic recoil using cardiovascular magnetic resonance myocardial feature tracking. PLoS ONE 9(10):e109164

24. Mancia G, Fagard R, Narkiewicz K, Redón J, Zanchetti A, Böhm M, Christiaens T, Cifkova R, De Backer G, Dominiczak A, Galderisi M, Grobbee DE, Jaarsma T, Kirchhof P, Kjeldsen SE, Laurent S, Manolis AJ, Nilsson PM, Ruilope LM, Schmieder RE, Sirnes PA, Sleight P, Viigimaa M, Waeber B, Zannad F, Task Force Members (2013) 2013 ESH/ESC Guidelines for the management of arterial hypertension: the Task Force for the management of arterial hypertension of the European Society of Hypertension (ESH) and of the European Society of Cardiology (ESC). J Hypertens 31(7):1281-1357

25. Li VWY, Cheung YF (2014) Right ventricular myocardial motion and deformation in adolescents and young adults after repair of coarctation of the aorta. Echocariography 32:797-804

26. Young AA, Cowan BR, Occleshaw CJ, Oxenham HC, Gentles TL (2002) Temporal evolution of left ventricular strain late after repair of coarctation of the aorta using 3D MR tissue tagging. J Cardiovasc Magn Reson 4(2):233-243

27. Di Salvo G, Pacileo G, Limongelli G, Verrengia M, Rea A, Santoro G, Gala S, Castaldi B, D’Andrea A, Caso P, Giovanna Russo M, Calabró R (2007) Abnormal regional myocardial deformation properties and increased aortic stiffness in normotensive patients with aortic coarctation despite successful correction: an ABPM, standard echocardiography and strain rate imaging study. Clin Sci 113(5):259-266

28. Jashari H, Lannering K, Ibrahimi P, Djekic D, Mellander M, Rydberg A, Henein MY (2016) Persistent reduced myocardial deformation in neonates after CoA repair. Int J Cardiol 221:886-891

29. Kimball TR, Reynolds JM, Mays WA, Khoury P, Claytor RP, Daniels SR (1994) Persistent hyperdynamic cardiovascular state 
at rest and during exercise in children after successful repair of coarctation of the aorta. JACC 24(1):194-200

30. Cook S, Ferketich A, Raman S (2009) Myocardial ischemia in asymptomatic adults with repaired aortic coarctation. Int J Cardiol 133:95-101

31. Menting ME, van Grootel RW, van den Bosch AE, Eindhoven JA, McGhie JS, Cuypers JA, Witsenburg M, Helbing WA, RoosHesselink JW (2016) Quantitative assessment of systolic left ventricular function with speckle-tracking echocardiography in adult patients with repaired aortic coarctation. Int J Cardiovasc Imaging 32(5):777-787

32. Kaplan NM, Silah JG (1964) The effect of angiotensin II on the blood pressure in humans with hypertensive disease. J Clin Invest 43:659-669

33. Parker FB, Farrell B, Streeten DH, Blackman MS, Sondheimer HM, Anderson GH Jr (1980) Hypertensive mechanisms in coarctation of the aorta. Further studies of the renin-angiotensin system. J Thorac Cardiovasc Surg 80(4):568-573

34. Pedersen TA, Pedersen EB, Munk K, Hjortdal VE, Emmertsen K, Andersen NH (2015) High pulse pressure is not associated with abnormal activation of the renin-angiotensin-aldosterone system in repaired aortic coarctation. J Hum Hypertens 29(4):268-273

35. Chiu HH, Chiu SN, Hu FC, Chen CA, Lin MT, Wang JK, Chang CI, Chen YS, Chiu IS, Wu MH (2009) Late cardiovascular complications after surgical or balloon angioplasty of coarctation of aorta in an asian cohort. Am J Cardiol 204:1139-1144

36. Bocelli A, Favilli S, Pollini I, Bini RM, Ballo P, Chiappa E, Zuppiroli A (2013) Prevalence and long-term predictors of left ventricular hypertrophy, late hypertension, and hypertensive response to exercise after successful aortic coarctation repair. Pediatr Cardiol 34(3):620-629
37. Ahmed MI, Desai RV, Gaddam KK, Venkatesh BA, Agarwal S, Inusah S, Lloyd SG, Denney TS Jr, Calhoun D, Dell'italia LJ, Gupta H (2012) Relation of torsion and myocardial strains to LV ejection fraction in hypertension. JACC Cardiovasc Imaging 5(3):273-281

38. Celic V, Tadic M, Suzic-Lazic J, Andric A, Majstorovic A, Ivanovic B, Stevanovic P, Iracek O, Scepanovic R (2014) Two- and three-dimensional speckle tracking analysis of the relation between myocardial deformation and functional capacity in patients with systemic hypertension. Am J Cardiol 113(5):832-839

39. Ikonomidis I, Tzortzis S, Triantafyllidi H, Parissis J, Papadopoulos C, Venetsanou K, Trivilou P, Paraskevaidis I, Lekakis J (2015) Association of impaired left ventricular twisting-untwisting with vascular dysfunction, neurohumoral activation and impaired exercise capacity in hypertensive heart disease. Eur J Heart Fail 17:1240-1251

40. Rodrigues JC, Amadu AM, Dastidar AG, Szantho GV, Lyen SM, Godsave C, Ratcliffe LE, Burchell AE, Hart EC, Hamilton MC, Nightingale AK, Paton JF, Manghat NE, Bucciarelli-Ducci C (2016) Comprehensive characterisation of hypertensive heart disease left ventricular phenotypes. Heart 102(20):1671-1679

41. Ayoub AM, Keddeas VW, Ali YA, El Okl RA (2016) Subclinical LV dysfunction detection using speckle tracking echocardiography in hypertensive patients with preserved LV ejection fraction. Clin Med Insights Cardiol 10:85-90

42. Drazner MH (2011) The progression of hypertensive heart disease. Circulation 123(3):327-334 